\title{
Prognostic Factors of Neurological Complications in Spinal Surgeries
}

\author{
Won Shik Shin, Dong Ki Ahn, Jung Soo Lee, Ki Hyuk Koo, In Seon Yoo \\ Department of Orthopedic Surgery, Seoul Sacred Heart General Hospital, Seoul, Korea
}

\section{Study Design: Retrospective study.}

Purpose: To determine prognostic factors of neurological complications (NCs) of posterior thoracolumbar surgeries.

Overview of Literature: There have been few reports on the prognosis of NCs according to the causes and treatment methods.

Methods: The subjects were 65 patients who had NCs for 19 years (1995-2013) after posterior thoracolumbar surgeries in Seoul Sacred Heart General Hospital. The degree of neurological injury was assessed using numeric scales as follows: G1, increased leg pain or sensory loss; G2, hemiparesis; G3, paraparesis; G4, cauda equine syndrome; and G5, complete paraplegia. The relative degree of neurological recovery was evaluated using four numeric scales as follows: Gr1, complete recovery; Gr2, almost complete recovery with residual sensory loss or numbness; Gr3, partial recovery with apparent neurological deficit; and Gr4, no recovery. The prognostic factors were investigated in terms of demographic and surgical variables that were available in a retrospective review.

Results: The causes were as follows: epidural hematoma (EH), 25 patients (38.5\%); insufficient decompression and fusion, 14 patients (21.5\%); mechanical injury, 11 patients (16.9\%); insufficient discectomy, four patients (6.2\%); and unknown, 11 patients (23.1\%). The grade of neurological injury was as follows: G1, 11 patients (16.9\%); G2, 34 patients (52.3\%); G3, 15 patients (23.1\%); G4, three patients (4.6\%); and G5, two patients (3.1\%). Thirteen patients received conservative treatment, and 52 underwent revision surgeries. Neurological recovery was as follows: Gr1, 21 patients (32.3\%); Gr2, 17 patients (26.2\%); Gr3, 20 patients (30.8\%); and Gr4, seven patients $(10.8 \%)$. The prognosis depended on the causes $(p=0.041)$. The subgroup analysis of the revision group revealed a significant correlation between the degree of neurological recovery and the timing of revision, irrespective of causes $(r=0.413, p=0.002)$.

Conclusions: The prognosis of NC depended on the causes. EH was the best and unknown was the worst prognostic factor. Revision should be performed as soon as possible for a better prognosis.

Keywords: Spinal surgery; Neurological complication; Treatment; Prognosis

\section{Introduction}

Neurological complication (NC) is a dreadful event that makes it difficult for surgeons to perform spinal surgeries and is often associated with technical problems. However, if the causes and clinical features are known, then these problems can be prevented and managed more appropriately. According to previous studies, the incidence of

Received Sep 15, 2017; Revised Nov 23, 2017; Accepted Nov 26, 2017

Corresponding author: Dong Ki Ahn

Department of Orthopedic Surgery, Seoul Sacred Heart General Hospital, 259 Wangsan-ro, Dongdaemun-gu, Seoul 02488, Korea

Tel: +82-2-966-1616, Fax: +82-2-968-2394, E-mail: adk0208@hanmail.net 
myelopathy or cauda equina injury in spinal surgeries was $0 \%-2.8 \%$ and the causes were surgical trauma, compression or distraction, ischemic injury, intradural or epidural hematoma (EH), mechanical compression by ligaments, intervertebral disc, and adjacent vertebral bodies [1-6]. However, it was difficult to extract studies on the influence of causes and treatment methods on the prognosis. Most previous studies have not explained various conditions and the corresponding remedies because these investigated NCs only in a specific disease entity. In addition, the real incidence of $\mathrm{NC}$ would be underestimated because they considered only the serious neurological deficit as an NC. In this study, we considered any trivial neurological symptom that did not preoperatively exist as an NC and investigated their treatments, final results, and prognostic factors to raise comprehensive awareness regarding the appropriate management of NCs.

\section{Materials and Methods}

In this retrospective study, the subjects were 65 patients who underwent posterior thoracolumbar surgeries during 19 years (1995-2013) and reported neurological symptoms that did not preoperatively exist before discharge after the index operations. In addition, their medical records and image tests were reviewed. The informed consents were waived. The causes were classified according to the initial and revision operation records, and the causes with no clear reason in the medical records and image tests were classified as "unknown." Neurological symptoms were evaluated at the initial maximum state and the final follow-up time. The degree of NC was assessed using five numeric scales as follows: G1, increased leg pain or sensory loss; G2, hemiparesis; G3, paraparesis; G4, cauda equina syndrome; and G5, complete paraplegia. Relative, rather than absolute, evaluation was used to assess the degree of improvement. The relative degree of neurological recovery was evaluated using four numeric scales as follows: Gr1, complete recovery; Gr2, almost complete recovery with residual sensory loss or numbness; Gr3, partial recovery with apparent neurological deficit; and Gr4, no recovery. Patients' age, sex, causes, the degree of NC, the degree of improvement, and whether revision or not and its timing were determined, and the factors influencing the final results were analyzed. In the assessment of prognostic factors, neurological recovery Gr1 and Gr2 were classified as good and Gr3 and Gr4 were classified as poor. Patients who received a revision surgery were assessed as a subgroup to evaluate the prognostic factors among them. In the statistical analysis, the Kruskal-Wallis test was used for neurological recovery according to the causes, and post-hoc analysis was performed using the Mann-Whitney $U$-test. Multivariable logistic regression analysis was conducted to evaluate prognostic factors and the relationship between the timing of revision and neurological recovery was analyzed using Spearman's correlation coefficient. The significance level was set at $p \leq 0.05$. All statistical analyses were performed using SPSS ver. 16.0 package (SPSS Inc., Chicago, IL, USA).

\section{Results}

Demographic data and variables of all patients were presented in Table 1.

\section{Causes}

The causes and number of patient were as follows: EH, 25 patients (38.5\%); insufficient decompression and fusion (IDF), 14 patients (21.5\%); mechanical injury (MI), 11 patients (16.9\%); insufficient discectomy (ID), four patients (6.2\%); and unknown, 11 patients (23.1\%) (Table 1).

\section{Treatment and recovery}

Thirteen patients were conservatively managed, and 52 underwent revision surgeries. The average timing of revision was 60 hours (range, 1-240 hours) from the end of the surgery. The average follow-up period was 23 months (range, 1-156 months). The grade of neurological recovery was as follows: Gr1, 21 patients (32.3\%); Gr2, 17 patients (26.2\%); Gr3, 20 patients (30.8\%); and Gr4, seven patients (10.8\%). The prognosis depended on the causes ( $p=0.007$ ) (Table 2), with a significant difference between $\mathrm{EH}$ and unknown in the post-hoc analysis $(p=0.001)$ (Table 3). Patients who underwent revision surgeries had better recovery than those who were conservatively managed $(p=0.023)$ (Table 2).

\section{Prognostic factors}

In the univariable analysis, both causes and treatment methods were significant prognostic factors; however, in the multivariable analysis, causes were the only significant 


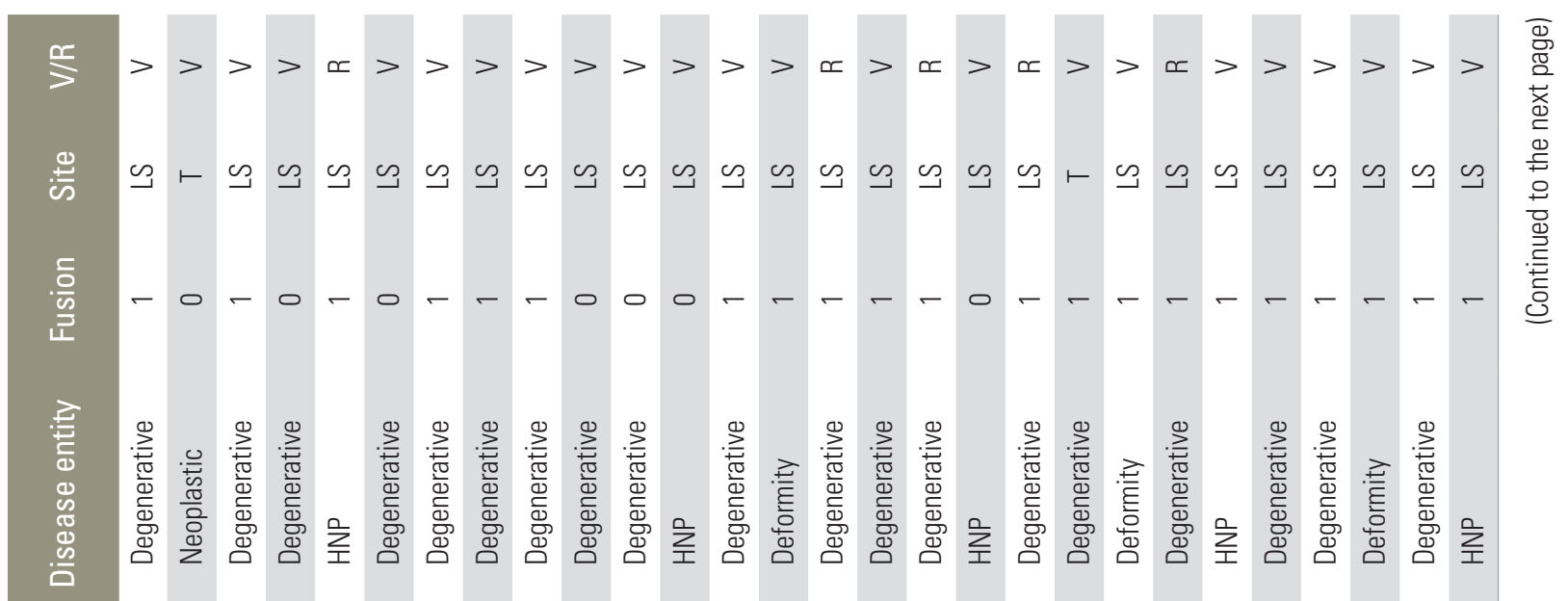

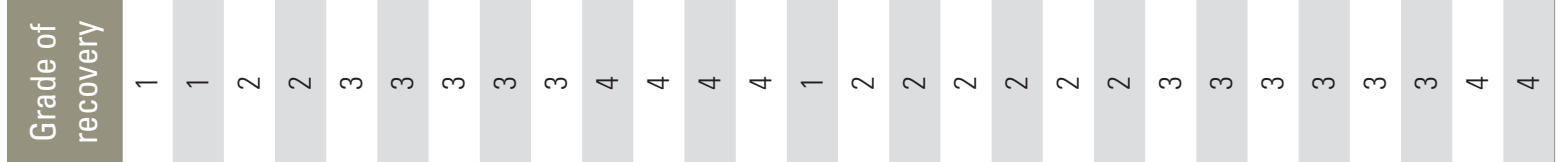

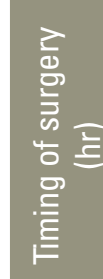

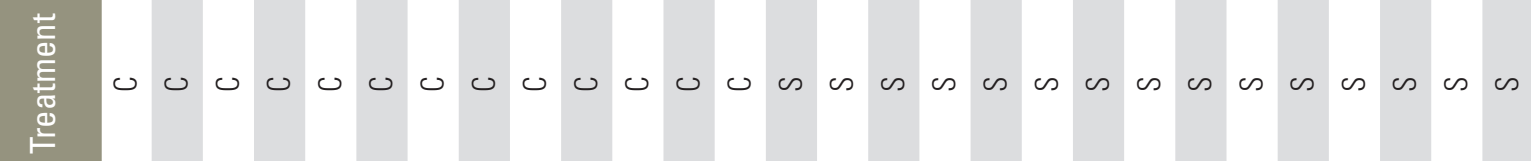

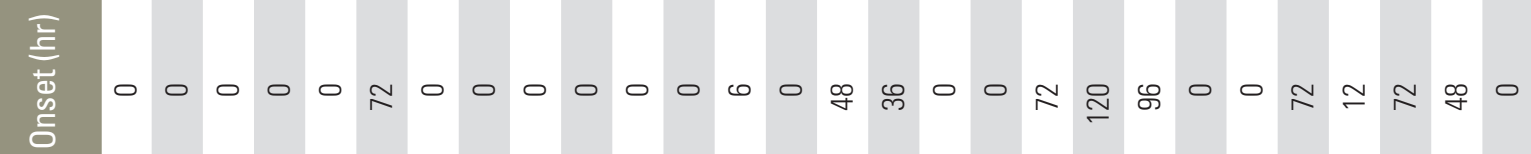

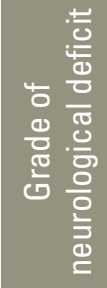

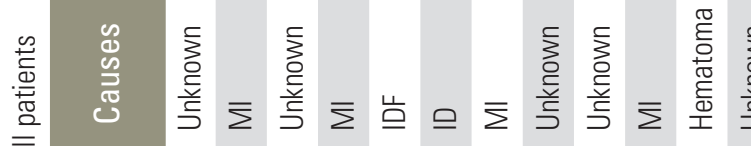

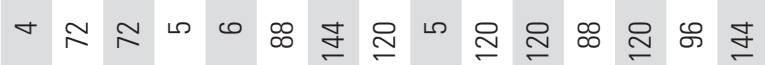
$\overline{\bar{\sigma}}$

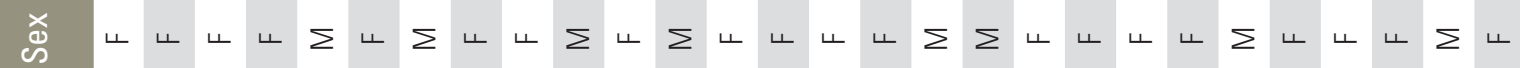




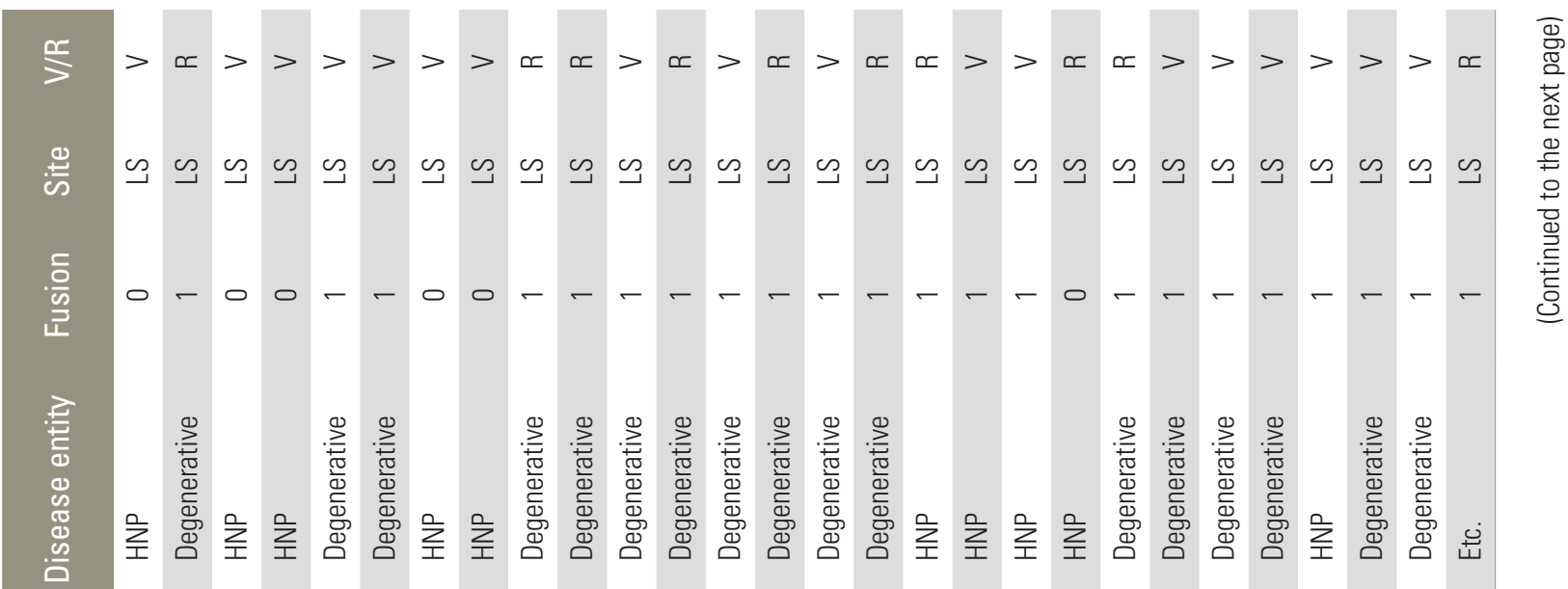

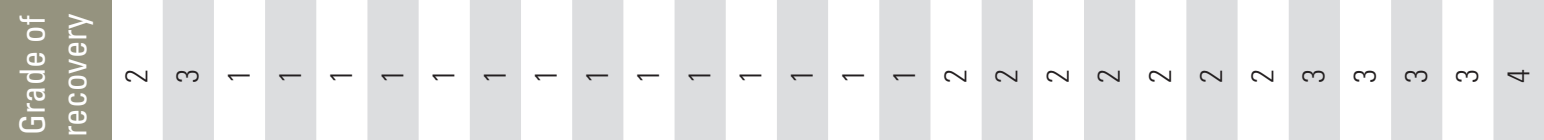

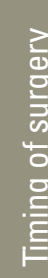

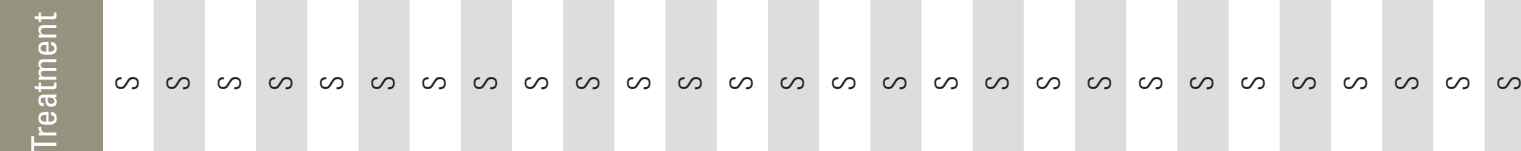



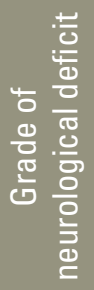

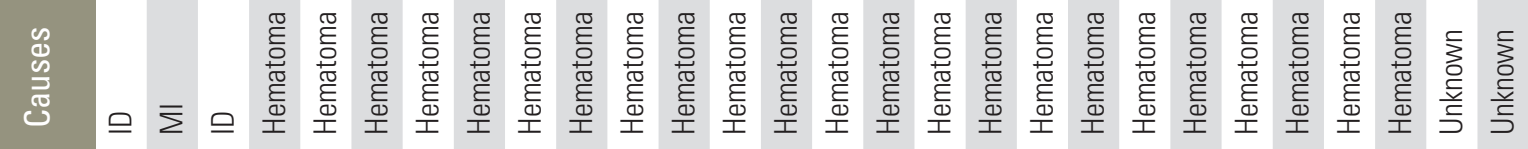

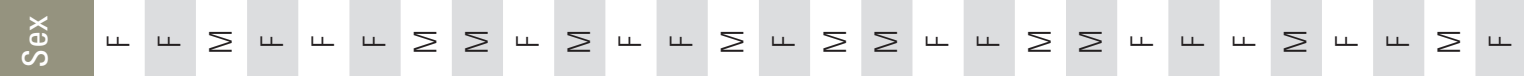

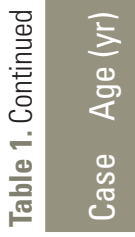

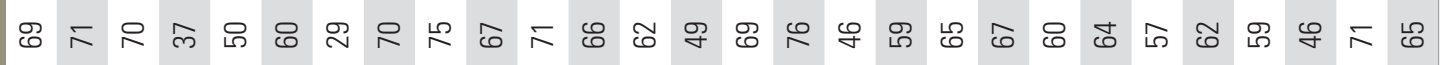
疋 

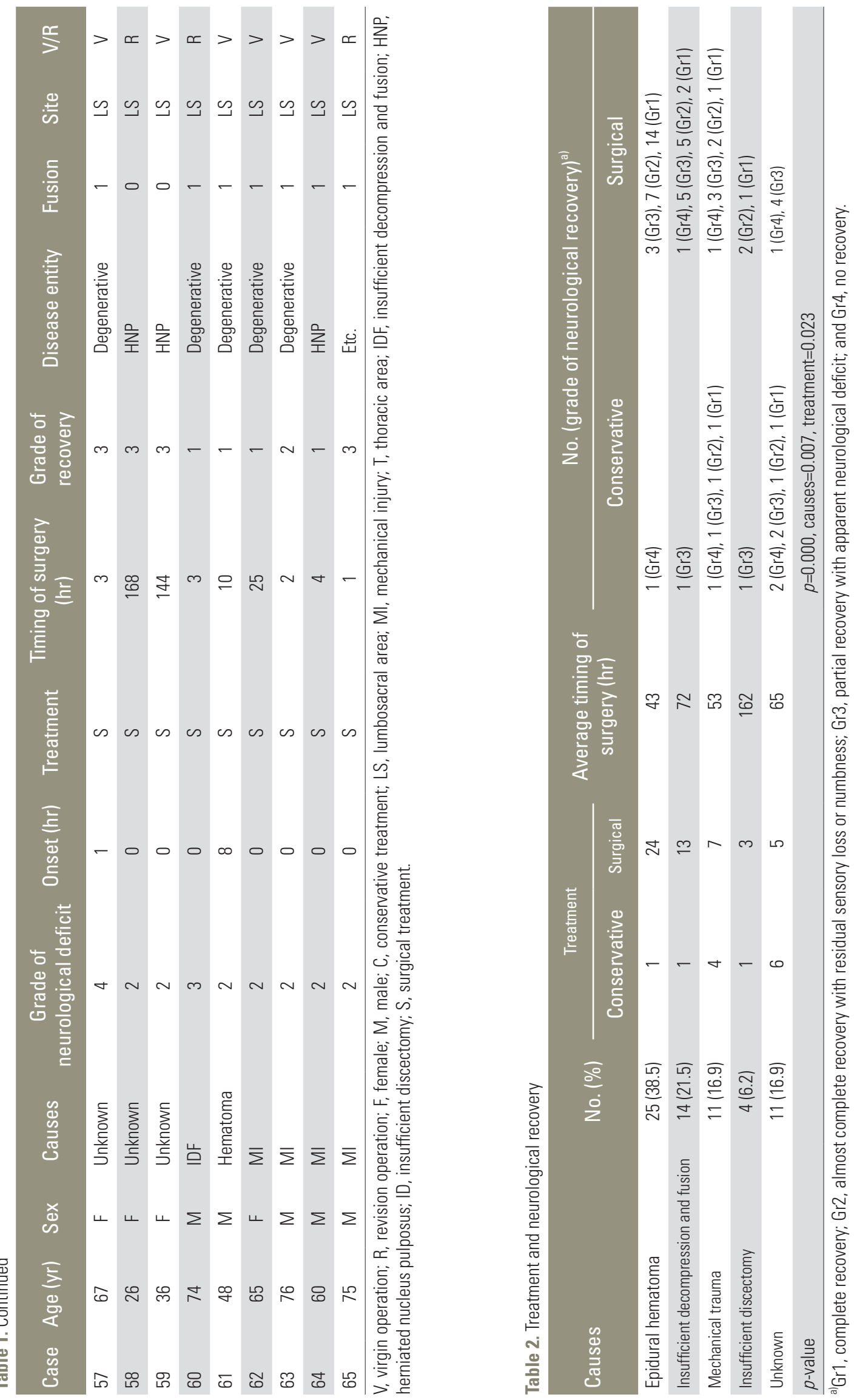
Table 3. Post-hoc analysis of neurological recovery according to the causes

\begin{tabular}{|c|c|}
\hline Causexcauses & $p$-value \\
\hline \multicolumn{2}{|l|}{ Epidural hematoma } \\
\hline Insufficient D\&F & 0.085 \\
\hline Insufficient discectomy & 0.948 \\
\hline Mechanical injury & 0.112 \\
\hline Unknown & 0.001 \\
\hline \multicolumn{2}{|l|}{ Insufficient D\&F } \\
\hline Epidural hematoma & 0.085 \\
\hline Insufficient discectomy & 0.921 \\
\hline Mechanical injury & 1.000 \\
\hline Unknown & 0.532 \\
\hline \multicolumn{2}{|l|}{ Insufficient discectomy } \\
\hline Epidural hematoma & 0.948 \\
\hline Insufficient D\&F & 0.921 \\
\hline Mechanical injury & 0.912 \\
\hline Unknown & 0.340 \\
\hline \multicolumn{2}{|l|}{ Mechanical injury } \\
\hline Epidural hematoma & 0.112 \\
\hline Insufficient D\&F & 1.000 \\
\hline Insufficient discectomy & 0.912 \\
\hline Unknown & 0.628 \\
\hline \multicolumn{2}{|l|}{ Unknown } \\
\hline Epidural hematoma & 0.001 \\
\hline Insufficient D\&F & 0.532 \\
\hline Insufficient discectomy & 0.340 \\
\hline Mechanical injury & 0.628 \\
\hline
\end{tabular}

Bold type is considered statistically significant.

D\&F, decompression and fusion.

factors (Table 4). In the subgroup analysis of 52 patients who underwent surgeries, the recovery did not depend on the causes ( $p=0.152$ ), and the timing of revision was the only prognostic factor $(p=0.015)$. Furthermore, there was a moderate correlation between the timing of revision and the degree of neurological recovery $(r=0.413, p=0.002)$.

\section{Discussion}

The management of NC in spinal surgeries warrants the identification of the causes and early management accordingly. This study showed that the most important prognostic factor was early surgical treatment. However, during the early postoperative period, advanced image tests are usually unavailable. Thus, initial management cannot help depending on surgeons' knowledge and expertise. In this study, EH was the most common cause and, at the same time, had the best prognosis. A patient who was conservatively managed reported relatively mild symptoms as G2. However, there was no improvement, although EH was completely resorbed in magnetic resonance imaging (MRI), which was performed 1 month postoperatively. In contrast, patients who underwent the surgical removal of EH exhibited better improvement despite severe neurological symptoms. Our results are consistent with those of Lawton et al. [7] reporting that the early removal of $\mathrm{EH}$ was the most important factor for neurological improvement. In the early period of this study, diagnosis and surgical removal was delayed because of the lack of awareness and expertise. In this study, three of four patients who underwent surgical removal after 4 days exhibited poor neurological recovery, whereas 14 of 16 patients who underwent surgical removal within 1day exhibited G1 recovery. All patients with EH used suction drains. Unfortunately, several previous studies have already established that EH cannot be prevented using a suction drain [8-10]; however, the reason behind this has not been reported. In our previous study, we presumed that the hypercoagulability of blood hampers the proper functioning of suction drains [11].

Revision surgeries of IDF tended to be delayed. Typically, the neurological symptom was vague during the immediate postoperative period and became distinct as time elapsed, particularly when walking began. It caused frequent delays in the accurate diagnosis and treatment. Conservative treatment was unsuccessful, and it showed poor results in $50 \%$. In case of instrumentation, the dimension of the spinal canal and neural foramen remains fixed. If lordosis increases, then the size of the spinal canal and neural foramen would decrease compared with that observed in the preoperative image tests. It necessitates a more comprehensive decompression. In the case of posterior lumbar interbody fusion, over reduction or an insufficient size of cages resulted in iatrogenic foraminal stenosis; although it was not easy to diagnose it using MRI because the neural foramen was hidden by a metal artifact, suspicion through clinical manifestations was cardinal.

Typically, MI displayed immediate symptom development. A patient injured by an osteotome showed gradual aggravation after 3 days. It was suspected to develop a 
Table 4. Prognostic factors and logistic regression analysis

\begin{tabular}{|c|c|c|c|c|c|}
\hline \multirow{2}{*}{ Variable } & \multirow{2}{*}{$\begin{array}{c}\text { Good } \\
(\mathrm{Gr} 1, \mathrm{Gr} 2)^{\mathrm{a})}\end{array}$} & \multirow{2}{*}{$\begin{array}{c}\text { Poor } \\
(\mathrm{Gr} 3, \mathrm{Gr} 4)^{\mathrm{a})}\end{array}$} & \multicolumn{2}{|c|}{$p$-value } & \multirow{2}{*}{$\begin{array}{c}\text { Odds ratio } \\
\text { (last reference) }\end{array}$} \\
\hline & & & Univariable & Multivariable & \\
\hline Age (yr) & $59.8 \pm 12.3$ & $60.2 \pm 11.1$ & 0.895 & & \\
\hline Sex (male/female) & $15 / 23$ & $9 / 18$ & 0.795 & & \\
\hline Onset (hr) & $10.2 \pm 23.6$ & $14.9 \pm 28.7$ & 0.488 & & \\
\hline Fusion/not & $29 / 9$ & $21 / 6$ & 1.000 & & \\
\hline Virgin/revision operation & $26 / 12$ & $21 / 6$ & 0.575 & & \\
\hline Site (lumbosacral/thoracic) & $36 / 2$ & $27 / 0$ & 0.507 & & \\
\hline$N$ grade $^{\text {bl }}$ & & & 0.420 & & \\
\hline G1 & 5 & 6 & & & \\
\hline $\mathrm{G} 2$ & 20 & 14 & & & \\
\hline G3 & 11 & 4 & & & \\
\hline G4 & 1 & 2 & & & \\
\hline G5 & 1 & 1 & & & \\
\hline Causes & & & 0.007 & 0.041 & \\
\hline Hematoma & 21 & 4 & & & 0.056 \\
\hline Insufficient decompression and fusion & 7 & 7 & & & 0.292 \\
\hline Insufficient discectomy & 3 & 1 & & & 0.085 \\
\hline Mechanical injury & 5 & 6 & & & \\
\hline Unknown & 2 & 9 & & & 0.292 \\
\hline Treatments (conservative/surgical) & $4 / 34$ & $9 / 18$ & 0.023 & 0.537 & 1.900 \\
\hline
\end{tabular}

Values are presented as mean \pm standard deviation or number, unless otherwise stated. Bold type is considered statistically significant.

${ }^{a} \mathrm{Gr}$, complete recovery; Gr2, almost complete recovery with residual sensory loss or numbness; Gr3, partial recovery with apparent neurological deficit; and Gr4, no recovery. ${ }^{b} \mathrm{G} 1$, increased leg pain or sensory loss; G2, hemiparesis; G3, paraparesis; G4, cauda equina syndrome; and G5, complete paraplegia.

compartment syndrome due to nerve root edema and partially improved by pedicle screw removal and pedicle excision to reroute the nerve root. Another patient injured by punch complained of neuropathic pain, which did not improve despite further decompression. Patients who were injured by a pedicle screw showed better recovery than those injured by other MIs. In addition, nerve compression by gelatin sponge was classified as an MI because its underlying mechanism was mechanical compression. Several case reports have presented similar experiences [12-15], commonly suggesting that gelatin sponge should be surgically removed, although the prognosis was not very favorable. Our patient showed immediate neurological symptoms, which kept increasing until revision was performed. Despite removal after 3 days, the result was poor. Thus, the mechanical pressure of gelatin sponge should be checked not at the time of insertion but after a while to enable its swelling.
If neurological symptoms were aggravated by ID, then the effect of conservative treatment was unpredictable. One of the three patients did not undergo revision surgery because the patient refused it, and the final result was poor. However, two of the three patients underwent revision surgery after 10 days of ineffective conservative treatment, and their final results were good. Finding similar situations in the published literature was challenging. However, we presumed that though the total volume of extruded nucleus pulposus was reduced by ID, nerve root edema and inflammatory reactions by surgical trauma might increase pain susceptibility. In addition, the average timing of revision surgery in ID was 162 hours. The reason for the delay was not difficulty in diagnosis but the expectation of improvement without revision surgery. In our limited cohort, the conservative treatment was not favorable in ID.

Devising any solution in patients with NCs with an un- 
known cause was challenging. We classified the cause as "unknown" if no specific cause was observed during the surgery and in the postoperative image tests and revision surgery. We examined five of 11 patients; however, the findings were nonspecific and the results were poor. Cramer et al. [4] have indicated that ischemic damage due to lower blood pressure during the surgery could be a cause. However, in our cohort, we could not determine any clue in the operation and anesthetic medical records supporting such a hypothesis.

There were some limitations in our study. First, we cannot affirm that all surgical conditions were consistent throughout the 19-year study period. During that time, our facility of MRI was changed once, we began using operating microscopes, and only one of five main operators performed surgeries throughout the study period. Second, because it was a retrospective study, we could not consider specific conditions of each case. In addition, because the incidence of NC was very low, the number of patients of each cause was not adequate to obtain statistically significant results. Finally, this was a review of a single hospital. Assumedly, a generalization of the authors' experiences may include several errors. We only proposed a possibility without any substantial evidence. However, the strength of this study was that no study has analyzed the final results regarding causes and treatment methods as prognostic factors for NC. In the future, we would like to present helpful data for the initial management of the variable causes of NC.

\section{Conclusions}

The final results of NC were influenced by causes. EH had the best and unknown had the worst prognosis. Among those who underwent surgical treatments, the earlier they had the revision surgeries, the better their prognosis was, irrespective of the causes. Therefore, performing a revision surgery as soon as possible on the exact diagnosis would be the best method to reduce the neurological deficit in NC of spinal surgeries.

\section{Conflict of Interest}

No potential conflict of interest relevant to this article was reported.

\section{References}

1. Antonacci MD, Eismont FJ. Neurologic complications after lumbar spine surgery. J Am Acad Orthop Surg 2001;9:137-45.

2. Bridwell KH, Lenke LG, Baldus C, Blanke K. Major intraoperative neurologic deficits in pediatric and adult spinal deformity patients. Incidence and etiology at one institution. Spine (Phila Pa 1976) 1998;23:324-31.

3. Bridwell KH, Lewis SJ, Edwards C, et al. Complications and outcomes of pedicle subtraction osteotomies for fixed sagittal imbalance. Spine (Phila $\mathrm{Pa}$ 1976) 2003;28:2093-101.

4. Cramer DE, Maher PC, Pettigrew DB, Kuntz C 4th. Major neurologic deficit immediately after adult spinal surgery: incidence and etiology over 10 years at a single training institution. J Spinal Disord Tech 2009;22:565-70.

5. Duncan JW, Bailey RA. Cauda equina syndrome following decompression for spinal stenosis. Global Spine J. 2011;1:15-8.

6. Yadla S, Malone J, Campbell PG, et al. Early complications in spine surgery and relation to preoperative diagnosis: a single-center prospective study. J Neurosurg Spine 2010;13:360-6.

7. Lawton MT, Porter RW, Heiserman JE, Jacobowitz R, Sonntag VK, Dickman CA. Surgical management of spinal epidural hematoma: relationship between surgical timing and neurological outcome. J Neurosurg 1995;83:1-7.

8. Chimenti P, Molinari R. Post-operative spinal epidural hematoma causing American Spinal Injury Association B spinal cord injury in patients with suction wound drains. J Spinal Cord Med 2013;36:213-9.

9. Walid MS, Abbara M, Tolaymat A, et al. The role of drains in lumbar spine fusion. World Neurosurg 2012;77:564-8.

10. Yi S, Yoon DH, Kim KN, Kim SH, Shin HC. Postoperative spinal epidural hematoma: risk factor and clinical outcome. Yonsei Med J 2006;47:326-32.

11. Ahn DK, Shin WS, Kim JW, Yi SM. Why Cannot suction drains prevent postoperative spinal epidural hematoma? Clin Orthop Surg 2016;8:407-11.

12. Alander DH, Stauffer ES. Gelfoam-induced acute quadriparesis after cervical decompression and fusion. Spine (Phila Pa 1976) 1995;20:970-1. 
13. Epstein NE, Silvergleid RS, Hollingsworth R. Increased postoperative cervical myelopathy and cord compression resulting from the use of Gelfoam. Spine J 2009;9:e19-21.
14. Friedman J, Whitecloud TS 3rd. Lumbar cauda equina syndrome associated with the use of gelfoam: case report. Spine (Phila Pa 1976) 2001;26:E485-7.

15. Herndon JH, Grillo HC, Riseborough EJ, Rich JC Jr. Compression of the brain and spinal cord following use of gelfoam. Arch Surg 1972;104:107. 